\title{
Cerebral Hemorrhage Following Chiropractic Activator Treatment - Case Report and Review of Literature
}

\author{
Fred L. Cohen ${ }^{1,2}$ \\ ${ }^{1}$ Private Practice of Neurological Surgery, Palm Beach Gardens, \\ Florida, United States \\ ${ }^{2}$ Gary Roberts and Associates, West Palm Beach, Florida, United States
}

Address for correspondence Fred L. Cohen, MD, JD, Gary Roberts and Associates, 580 Village Boulevard, Suite 300, West Palm Beach, FL 33409, United States (e-mail: vannefred@aol.com).

J Neurol Surg Rep 2016;77:e162-e167.

\begin{abstract}
Keywords

- activator

- chiropractic

- stroke

- trauma

- cerebral hemorrhage

Background Despite widespread utilization of chiropractic treatment for various ailments, there is a paucity of documentation regarding intracerebral hemorrhage related to chiropractic trauma. Stroke has been reported from cervical manipulation, although with a suggested low incidence. Activator treatment, an instrument that produces a high-velocity, low-amplitude impact to the spine, is considered especially safe. There are no prior reports of intracerebral hemorrhage resulting from a chiropractic activator treatment.

Case Description A 75-year-old woman was admitted to the hospital with a history of headaches, visual difficulties on the right, and speech disturbance of relatively acute onset. CT scan showed a brain hemorrhage in an unusual location. Extensive evaluation was undertaken because this was thought to be a spontaneous event. No cause was found on imaging. Subsequent history revealed a chiropractic activator treatment applied directly to the junction of the back of her head and the upper cervical spine immediately prior to the onset of symptoms. Her clinical course is described.

Conclusions This appears to be the first report linking traumatic intracerebral hemorrhage with a chiropractic activator treatment. The use of this modality in an elderly population, with widespread utilization of anticoagulants and platelet inhibitors, is of potential concern.
\end{abstract}

\section{Introduction}

An elderly but very active woman with a long history of pain complaints, including neck pain, and prior chiropractic adjustments, developed a headache-based acute illness with speech and visual complaints shortly after the application of an activator treatment in which the instrument was directed by the chiropractor at the base of her skull on the left side. A subsequent $\mathrm{CT}$ scan of the brain without contrast showed a hemorrhage with acute characteristics consistent with the time from the treatment and very localized in this precise location. Several experienced and senior neurosurgeons caring for her considered this an unusual location for a hemorrhage of this type, regardless of cause, and certainly not from any of the usual causes or types of trauma routinely associated with a busy and active emergency room and trauma service. This led to a fairly extensive workup, in part because initially there was no history of any conventional trauma, and this was first considered a spontaneous hemorrhage. No underlying cause was found. The current literature on activator treatment and complications appeared to be sparse on complications in general and nonexistent specifically on a complication of this type. This report describes her presentation, the evolution received

June 30, 2016 accepted after revision November 7, 2016
DOI http://dx.doi.org/ $10.1055 / \mathrm{s}-0036-1597626$. ISSN 2193-6358.

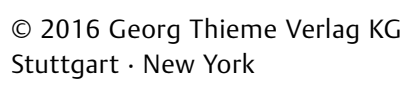

License terms

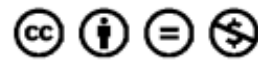


of her imaging studies, and her outcome. It then briefly reviews the history, theories and mechanisms of action, and prior reported complications of activator treatment. It is suggested that these events were initiated by a single activator treatment.

\section{Case Report}

A 75-year-old active woman, still working regularly, presented to a local hospital emergency room in the spring of 2013 with a 3-day history of the acute onset of severe left temporal headache, initially self-treated with nonsteroidals, to which they were resistant. Additional complaints included some vague right eye blurring of vision and a mild speech disturbance. Her primary-care physician ordered an outpatient MRI, which was interpreted as showing a small subacute left posterior temporal lobe hemorrhage and referred her to the emergency room where she was categorized as a "stroke alert" and evaluated according to the hospital "stroke-alert" protocol. There was no prior history of migraine, but some mild treated hypertension. Initial brain CT ( - Fig. 1) showed a fairly localized intracranial hemorrhage in the left occipital area. Tele-neurology (on-call) recommended hospital admission after a CT angiogram showed no underlying vascular cause. Neurology and neurosurgery were consulted. She subsequently gave a history of chronic neck and back pain, but no headache, for which she had intermittently over some years received traditional chiropractic adjustments. Her current "illness" started with a "mental status" change right around the time of an activator treatment to the base of the left side at the junction of the skull with the upper cervical spine. She became concerned enough a few days later, because of the persistence of unremitting headache, to contact her primary-care physician. This history was compatible,

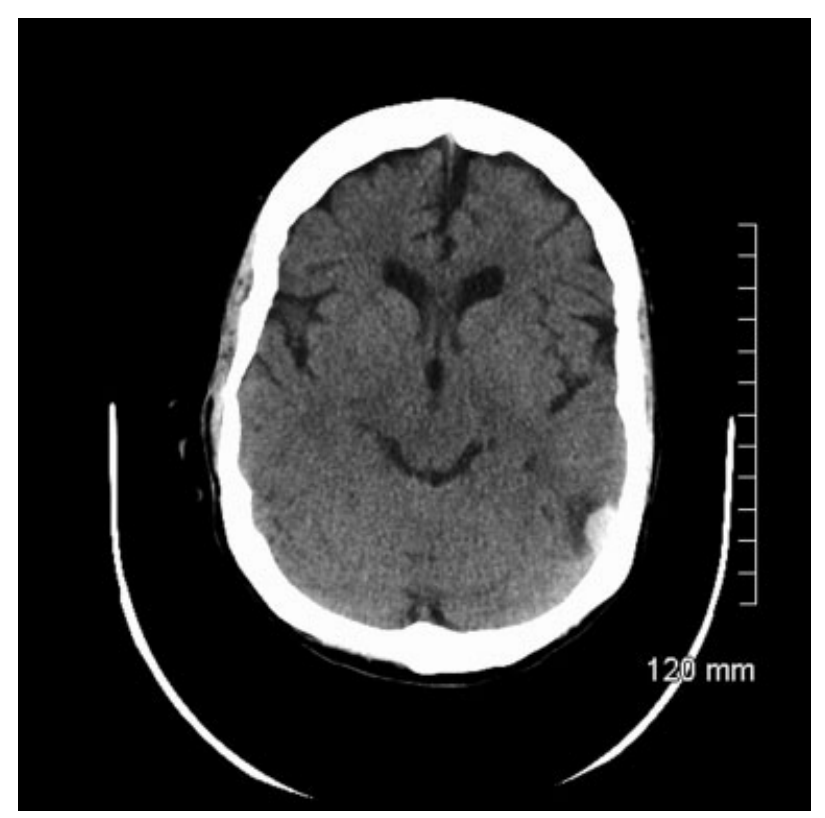

Fig. 1 CT scan of the brain in axial projection following admission showing this very unusual location for any type of hemorrhagic abnormality, requiring better definition as to anatomic location. in retrospect, with the timing and the site of application of the activator treatment.

There was some difference of opinion even among the neurosurgeons and neurologists who consulted as to whether this hemorrhagic abnormality was extra-axial or intraparenchymal. She underwent CT angiography, MR angiography, and ultimately 4-vessel cerebral angiography because of the concern regarding both the location of the lesion and the etiology. No literature connected this presentation with activator treatment. Ultimately, it was determined (based on MRI characteristics) to be primarily subarachnoid and displacing but not involving any brain parenchyma, and without any extra-axial component (-Fig. 2A-C). From the CT scan alone, neither the neuroradiologists nor the senior neurosurgeons could be certain this wasn't primarily or all extra-axial. The MRI was extraordinarily helpful from multiple points of view: in determining that it was primarily subarachnoid and not extra-axial or intraparenchymal (thus making it unlikely to have been the result of a hematoma or cryptogenic infarction/hemorrhage); in determining that it was more occipital than temporal; in determining that there was no underlying infarction (and therefore not the result of a small-vessel lacunar infarction). She was not taking any anticoagulants or antiplatelet agents and had a relatively unremarkable past medical and surgical history. Although she did not have a formal visual field examination or an ophthalmology consultation, she was found to have an incomplete right homonymous hemianopsia on clinical exam by the neurologist.

After a 4-day hospitalization for evaluation and observation, she was discharged, neurologically improved in terms of visual and speech symptoms as well as headache complaints, to outpatient follow-up. She has remained well with resolution of imaging abnormalities and no reoccurrence of symptoms.

\section{Discussion}

Patients seen through our emergency room are divided into traumatic and nontraumatic (spontaneous) brain hemorrhages. The latter category includes stroke alerts. Most patients with brain hemorrhage from traumatic etiologies do not undergo the same extensive imaging evaluation as do the spontaneous hemorrhage patients. There is, additionally, less concern in the traumatic cases that there will be a delayed, secondary, or subsequent hemorrhage from an underlying and/or unrecognized problem. Initially, this woman did not give a typical history for any brain trauma, which would have led to a less exhaustive evaluation. After a thorough search for an underlying cause for her hemorrhage, additional history revealed the activator treatment, but a literature search provided no insight as to reports of hemorrhagic complications resulting from activator treatments.

Although a full discussion of activator history is beyond the scope or intent of this article, a brief review will likely be helpful to the reader, many of whom may have little to no familiarity with this fairly common form of chiropractic treatment. 

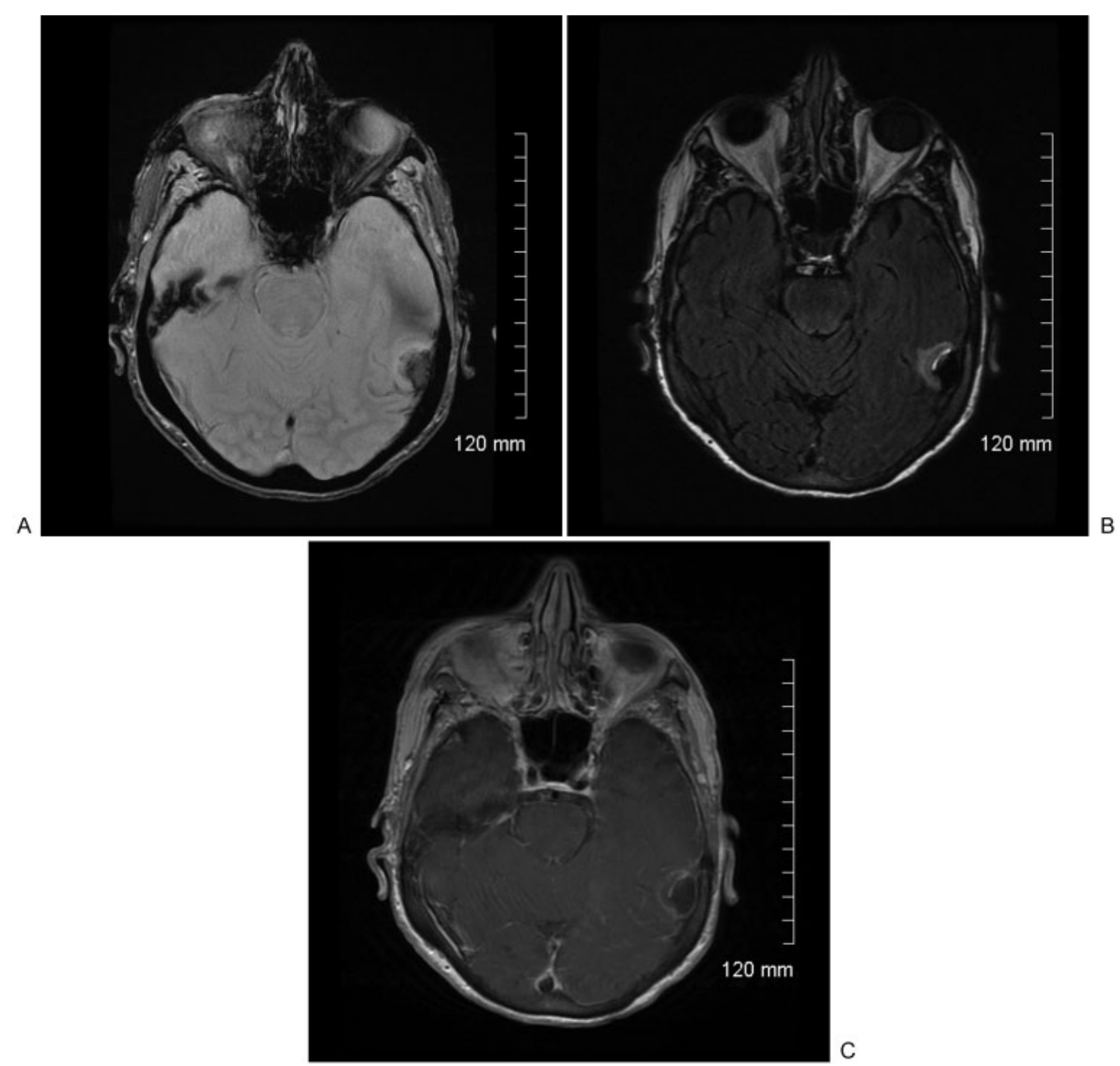

Fig. 2 MRI scan in (A) GRE, (B) T2 Flair as well as (C) T1 with contrast acquisitions, together showing the anatomic location of this hemorrhage far anterior in the subarachnoid space of the occipital lobe but displacing brain parenchyma and surrounded by an area of edema and encephalomalacia, an usual location for any type of hemorrhagic abnormality.

The formal chiropractic era dates to Dr Daniel David Palmer in 1895 with the opening of the Palmer School of Chiropractic (still in existence and active today, over 100 years and many scientific challenges later). This was followed by Dr Willard Carver who opened a competing school. Carver believed that other treatments were needed beyond just spinal manipulation. The Palmer devotees are "straight" chiropractors. Carver followers are "mixed" chiropractors. ${ }^{1,2}$

Up to the time of Dr Arlan Fuhr in 1967, there were two main forms of chiropractic spinal techniques. The first and most well known is the chiropractic manipulation or HVLA (rapid [High-Velocity], short\{Low Amplitude\} thrusts). This produces the classic "crack" associated with chiropractic maneuvers in the minds of many laymen. The other is spinal mobilization, a more gentle application of force (low velocity, high amplitude). Fuhr, in devising the first activator instrument in rural Minnesota in association with Dr Warren C. Lee in 1967, added an additional method to perform manipulation. ${ }^{1}$ Since then it has gone through various refinements and evolution from its first patent in 1978 as an Activator Adjusting Instrument (manufactured by Union Broach in New Jersey), Activator II in 1994, the Activator Signature, the Air Activator (a compressed-gas instrument), the Impulse from Neuromechanical Innovations, an electromechanical instrument) the battery-powered and cordless Activator V. ${ }^{1,3,4}$ Aside from the instrument itself (a mechanical adjusting instrument), a separate and equally controversial assessment method developed in which the location or spinal segment at which the activator instrument is applied is determined by the use of functional leg length inequality measurements 
made with the patient prone. ${ }^{1}$ With the addition of the activator (instrument and method), there are now basically three sorts of chiropractic treatment provided. The activator is in the category of Mechanical Adjusting Devices. ${ }^{4,5}$

The activator instrument itself is a small handheld springloaded instrument, which delivers an impulse to the spine. Ideally, the activator spring tension is supposed to be loaded to an amplitude commensurate with the bone size and density that it is intended to adjust. ${ }^{6}$ Considered to be a high-velocity (energy) low amplitude (light/low-force) stimulus in the category of a manipulation, there have been many articles devoted to the physics of the device. In general, it gives off $\sim 0.3$ Joules of kinetic energy in a 3 millisecond pulse. ${ }^{4}$ What is far less available and less researched are serious complications of activator treatment. This is underscored if one considers the widespread adoption of these techniques and estimated frequency of their use in the population of patients who visit and treat with chiropractors. Consider that the activator method was first offered as an elective course at Logan College in 1980, added to the Core Curriculum of Parker College in 1985. ${ }^{1}$ The Fall 2014 course offering brochure for activator methods lists 12 major U.S. and Canadian cities where instruction is offered. ${ }^{7}$ It has been variously estimated that $69.9 \%$ of chiropractors use the activator technique and $23.9 \%$ of patients received it. Forty-five thousand chiropractors worldwide use some form of activator instrument or technique. ${ }^{6}$ In 1998 , the AMA estimated $11 \%$ of the U.S. population visited a chiropractor in the previous year. With a U.S. population estimated to be 288 million, that is 31.6 million people. If only $23.9 \%$ of this number receives activator treatment of some sort, it still represents some 6.9 million patients.

The effectiveness of activator treatment is likewise beyond the scope of this paper, except to say that it has been and remains both controversial and contentious. ${ }^{2,3,8,9}$ Scientific accuracy has always been difficult to achieve in the area of chiropractic "science" and "outcomes." In this regard, Dr Fuhr established the National Institute of Chiropractic Research, leading to a $1985 \mathrm{NIH}$ grant for chiropractic research to study the safety and effectiveness of the activator. The results of this study (a randomized controlled trial) were presented in Orlando, FL, in March of 2014. Here, activator treatment fared worse than Manual Manipulation (and so did "usual medical care.") ${ }^{1,10-12}$ Wood found both the Activator II and manual manipulation to be equally effective in the cervical spine. ${ }^{13}$

Safety concerns have always existed, especially in the medical community, regarding chiropractic care. In the stopped randomized trial reported by Gemmeli and Miller, adverse events appear to be distinguished from serious complications. Adverse events are transient side effects, all self-limited and of short duration (1-3 days) and all characterized as mild, in the form of: increased neck pain; radiating pain; arm weakness; arm numbness; headache; fatigue; dizziness; muscle twitching. Of 47 patients enrolled (16 activator, 16 manipulation, 15 mobilization), the activator group had more of these adverse events than either of the other groups $\left(21,14\right.$, and 8 , respectively). ${ }^{14}$ The reoccurring and overriding concerns have been for stroke associated with, or the result of, manual manipulation of the cervical spine. ${ }^{15-22}$ These are serious complications. Considering the admonitions, it turns out that stroke secondary to chiropractic cervical manipulation is reported to be exceedingly rare. NYU Langone Medical Center cites almost 200 serious reported complications, stroke among them, associated with neck manipulation. Attempting to put this complication in perspective, the article goes on to estimate 1 complication per 1 million individual treatment sessions. With cervical manipulation involving a course of treatment, the rate of stroke is $1 \mathrm{in} 100,000$. The rate of death is one in 400,000 . The death rate from NSAIDs among people using them for arthritis is 4 in 10,000. This makes chiropractic neck manipulation 100 to 400 times safer than regular NSAID use. ${ }^{23}$ Even rarer, according to many publications, are complications related to activator treatment. A recent article in "The Chiropractic Report" from May 2014 notes activator use especially in children, patients with osteoporotic bone fragility, and those fearful of manipulation because of both its effectiveness and its safety. ${ }^{1}$ Other authors mention children in relation to activator safety. ${ }^{24,25}$ Thiel and colleagues in 2007 reported on neck manipulation in 19,722 patients comprising 28,807 treatment consultations and 50,276 cervical spine manipulations. In this study group, there were no reports of serious adverse events. There were some minor side effects. ${ }^{22}$ Nykoliation and Mierau reported details of three cases of adverse effects potentially associated with the use of mechanical adjusting devices. Two of the three cases were summarized from medical malpractice actions that resulted from the care. Only one of the three suffered a completed stroke. The other two had nothing to do with the brain or vascular issues. Fuhr (inventor of the activator), commenting on this article, notes that "causal attribution of harm from AAI adjustments could not be made in these reports." “.....recent reviews suggest that CVIs and CVAs are rare, random, and unpredictable, and possibly independent of treatment." In 1995, Carey reported on 13 strokes in Canada between 1986 and 1990, 12 of which resulted in malpractice claims, alleging the CVA was a result of chiropractic treatment. None mentions the activator. Carey claimed the accurate incidence rate for CVAs in chiropractic practice is about one occurrence in every 3-3.8 million manipulations. ${ }^{20}$

Virtually, every article in which frequency of serious adverse events is discussed hedges on the accuracy and reliability of the data and the conclusion. This is underscored by the fact that Haldeman analyzed 64 cases of cerebrovascular accident after spinal manipulation. Although he reported on them in the Journal of Neurology in 2002, these are cases that had been referred to him over the previous 16 years for medico-legal review and, during that time, none of them had been reported in the medical literature. Similarly for Hansis, who published in 2004 in the orthopedic literature on 57 patients who had been referred to a German Medical Council during a 28 -year period, but none reported in the literature. Underreporting overall seems fairly concerning. ${ }^{26}$

The most exhaustive study of safety and adverse effects and referencing multiple other articles was done by Sutcliffe et al. ${ }^{24}$ They summarized and analyzed seven systematic 
reviews, four retrospective/prospective cohort studies, one case series, and two cross-sectional surveys. However, there is no specific mention of activator treatment anywhere in this article or analysis. The only intracranial hemorrhage mentioned is incidental subarachnoid hemorrhage in association with vertebral artery injuries with associated aneurysm, dissection, ischemic infarction injuries. In the majority of those cases ( $60 \%$ or so) the bleeding is in the subarachnoid space. In the patient reported here, all four of the extracranial vessels were imaged, all were normal, and there was no evidence of underlying infarction. Vertebral artery dissection and vertebro-basilar stroke are themselves rare events whose causal connection to chiropractic adjustments has been questioned but not definitely established. A small number of cases have reported on spinal epidural hemorrhage/hematoma related to chiropractic treatments. ${ }^{16}$ These are summarized by Huang and colleagues in 2015. A careful analysis of all of the imaging studies done in this case, while initially interpreted as epidural hemorrhage, was ultimately determined to be focal subarachnoid blood displacing but not involving the parenchyma of the far-anterior occipital lobe, and consistent with the time course of her illness and the application of the activator stimulus.

Causation is a major concern here. It is extremely difficult to prove specific causation in medical and biological systems under the best of circumstances. It is frequently inferred by epidemiological reasoning or evidence. ${ }^{27,28}$ While there are other potential causes of the hemorrhage that occurred in this case, none is as or more likely than the activator stimulus. In support of the activator as the cause of the hemorrhage, the symptoms began almost immediately after the activator treatment (a temporal relationship), the area to which the activator was applied is almost directly superficial to the area of hemorrhage (a spatial relationship), the anatomic location of this hemorrhage is statistically unusual for any underlying and/or preexisting conditions, including stroke. The MRI confirms that there was no infarction underlying the area of hemorrhage. The MRA disclosed no dissections or vascular lesions present. The only mechanisms left are trauma or cryptic vascular lesion that ruptured, obliterated itself, and occurred coincident to the activator stimulus. Although Activator stimulus is not high energy, it nonetheless in this case was targeted to the cervico-occipital junction, an area where neural tissue is among the most vulnerable and least protected and closest to the skin (as opposed to the lower cervical or any of the thoracic or lumbar spine). There are many articles that make reference to minor or trivial head injury as a likely cause of intracranial hemorrhage. ${ }^{29-33}$ Difficulty in linking causality to a chiropractic treatment event is underscored by the fact that there has never even been any real proof that chiropractic manipulation is the cause of vertebral artery dissection and/or occlusion. Despite this, the literature reflects relatively widespread agreement or consensus regarding this link (see Fuhr ${ }^{4}$ this article). In this case, while the lack of association (strength) between activator treatments and any serious complications is exactly what makes it reportable and of interest, it is also the exact reason why causation cannot be either proven or more strongly correlated. In the absence of reporting suggested links of this type, any latent or even obvious activator complications will never reach the level of awareness or knowledge.

\section{Conclusions}

The author was unable to find a single documented case in which a brain hemorrhage in any location was reported from activator treatment. As such, this case appears to represent the first well-documented and reported brain hemorrhage plausibly a consequence of activator treatment. In the absence of any relevant information in the chiropractic or medical literature regarding cerebral hemorrhage as a consequence of activator treatment, this case should be instructive to the clinician who is faced with a diagnostic dilemma and should not forget to inquire about activator treatment as a potential cause of this complication. Our case had a benign course, but we do not rule out a more serious or potentially dangerous clinical course or adverse outcome. This is of heightened concern in the elderly and/or those with treatment-induced coagulopathy or platelet inhibition.

In light of all of the difficulties inherent in linking chiropractic treatments, including activator treatments, with serious neurological events, it is very possible that intracranial hemorrhage is far more frequent than reported. Several articles comment on the likelihood that complications of this type are almost certainly underreported. Most of the incidents mentioned in case series or surveys had never been previously reported. Neurologists, neurosurgeons, and chiropractors should be more vigilant both in the application and evaluation of these methods in all patients who report new neurologic-type symptoms following a manipulation (including an activator application) to the occiput or the cranio-cervical junction.

\section{Note}

The author, a practicing neurological surgeon and also a practicing attorney, has not had any personal experience litigating, representing, or defending any cases involving chiropractic complications of stroke or hemorrhage.

\section{References}

1 Activator methods, the rise and new status of instrument adjusting. In: Chapman-Smith D, ed. The Chiropractic Report. 28(3), May 2014, pp. 1-8

2 Chiropractic. Crouse Hospital, Syracuse, New York, found at http:// www.crouse.org/health/tnp/theraphy/chiro/

3 Aetna Clinical Policy Bulletin/Chiropractic Services. Number: 0107, found at http://www.aetna.com/cpb/medical/data/100_199/0107. html

4 Fuhr AW, Menke JM. Status of activator methods chiropractic technique, theory, and practice. J Manipulative Physiol Ther 2005;28(2):e1-e20

5 Pearson SH. The Dark Side of Chiropractic. Saturday, February 27, 2010, found at http://pearsonpayload.blogspot.com/2010/02/ dark-side-of-chiropractic.html

6 Activator Technique in Wikipedia, the free encyclopedia, found at http://en.wikipedia.org/wiki/Activator_technique 
7 Activator Seminar Schedule. Spring 2016, found at https:// d3o0u642xgsij.cloudfront.net/wp-content/uploads/2015Fall2016Spring-Schedule

8 Activator Fares Worse than Manual Manipulation in New Large Scale RCT Study, in Neuromechanical Innovations newsletter, found at http://www.neuromechanical.com/activator_fares_worse/

9 Archive for Category Activator Methods Chiropractic Technique. General Articles, found at https://www. activator.com/category/ activator-merhods-chiropractic-technique-general-articles/

10 Cleveland Chiropractic College. sponsor. Comparison of Activator Manipulation Versus Manual Side Posture Manipulation in Patients with Low Back Pain. NIH study found at ClinicalTrials.gov, identifier NCT00487861. URL https://clinicaltrials.gov/ct2/show/NCT00497861

11 Schneider MJ, et al. A Comparison of Spinal Manipulation Methods and Usual Medical Care for Low Back Pain: A Randomized Clinical Trial. Proceedings of the 2014 ACC RAC Conference, Orlando, Florida, March 20-22, 2014, found at www.chiroaccess.com/News/ACC-RACOrlando-Fl-March-20-22-2014-The-Premier-Annual-Scientific-Conference-of-the-Chiropractic-Porfession.aspx?id=0000543

12 Schneider M, Haas M, Glick R, Stevans J, Landsittel D. Comparison of spinal manipulation methods and usual medical care for acute and subacute low back pain: a randomized clinical trial. Spine 2015;40(4):209-217

13 Wood TG, Colloca CJ, Matthews R. A pilot randomized clinical trial on the relative effect of instrumental (MFMA) versus manual (HVLA) manipulation in the treatment of cervical spine dysfunction. J Manipulative Physiol Ther 2001;24(4):260-271

14 Gemmett H, Miller P. Relative effectiveness and adverse effects of cervical manipulation, mobilization and the activator instrument in patients with sub-acute non-specific neck pain: results from a stopped randomized trial. Chiropractic \& Osteopathy 18(20) 2010. Also found at http://www.chiromt.com/content/18/1/20

15 Carey PF. Cerebral vascular accidents: a report on the occurrences and the incidence in a 5. year period in Canada. J Can Chiropr Assoc 1995;39(2):94-95

16 Huang M, Barber SM, Moisi M, et al. Cervical epidural hematoma after chiropractic spinal manipulation therapy in a patient with an undiagnosed cervical spinal arteriovenous malformation. Cureus 2015;7(8):e307

17 Hurwitz EL, Morgenstern H, Vassilaki M, Chiang LM. Frequency and clinical predictors of adverse reactions to chiropractic care in the UCLA neck pain study. Spine 2005;30(13):1477-1484

18 Jones J, Jones C, Nugent K. Vertebral artery dissection after a chiropractor neck manipulation. Proc Bayl Univ Med Cent 2015; 28(1):88-90
19 Klougart N, Leboeuf-Yde C, Rasmussen LR. Safety in chiropractic practice. Part II: Treatment to the upper neck and the rate of cerebrovascular incidents. J Manipulative Physiol Ther 1996; 19(9):563-569

20 Nykoliation J, Mierae D. Adverse effects potentially associated with the use of mechanical adjusting devices: a report of three cases. J Can Chiropr Assoc 1999;43(3):161-167

21 Rothwell DM, Bondy SJ, Williams JI. Chiropractic manipulation and stroke: a population-based case-control study. Stroke 2001;32(5): 1054-1060

22 Thiel HW, Bolton JE, Docherty S, Portlock JC. Safety of chiropractic manipulation of the cervical spine: a prospective national survey. Spine 2007;32(21):2375-2378, discussion 2379

23 NYU Langone Medical Center. Chiropractic, found at http://www. med.nyu.edu/content?ChunkID=37431

24 Sutcliffe P, Clar C, Tsertsvadze A, et al. Clinical and cost-effectiveness of manual therapy for the management of a variety of musculoskeletal and non-musculoskeletal conditions: a systematic review and narrative synthesis. (pp 1-336) found at https:// www2.warwick.ac.uk/fac/med/research/hscience/pet/reportforcollegeofchiropractors/manualtherapyreport_201112_final.pdf

25 Weil A. Chiropractic Therapy. , found at http://www.drweil.com/ drw/u/ART02024/Chiropractic-Therapy.html

26 Ernst E. Adverse effects of spinal manipulation: a systematic review. J R Soc Med 2007;100(7):330-338

27 Uhrenholt L, Freeman MD, Webb AL, Pedersen M, Boel LWT. Fatal subarachnoid hemorrhage associated with internal carotid artery dissection resulting from whiplash trauma. Forensic Sci Med Pathol 2015;11(4):564-569. doi:10.1007/s12024-015-9715-3

28 Freeman MD, Zeeger MP, eds. Forensic Epidemiology: Principles and Practice. Academic Press: Eslevier; 2016:416 pp.

29 Agamanolis DP. Neuropathology: An Illustrated Interactive Course for Medical Students and Residents. Chapter 4 (Traumatic Brain Injury and Increased Intracranial Pressure), found at http://neuropathology-web.org/chapter4/chapter4aSubdurakepidural.html.

30 Adhiyaman V, Asghar M, Ganeshram KN, Bhowmick BK. Chronic subdural haematoma in the elderly. Postgrad Med J 2002;78(916): 71-75

31 Head Injuries, found at http://www,surgiwiki.com/wiki/Head_injuries

32 Weisberg LA, Garcia C, Strub R. Essentials of Clinical Neurology: Head Trauma. (Chapter 12) www.psychneuro.tulane.edu/neurolect/

33 Dacey RG Jr, Alves WM, Rimel RW, Winn HR, Jane JA. Neurosurgical complications after apparently minor head injury. Assessment of risk in a series of 610 patients. J Neurosurg 1986;65(2): 203-210 